\section{FOLDING ESMARCH ANESTHESIA MASK.}

VICTOR F. MARSHALL, M.D. APPLETON, WIS.

The accompanying illustrations show a modification I have made of Esmarch's anesthetic mask. The hoop wires are supplied with joints at the ring base, thus allowing the hoops to be folded on each other. This gives

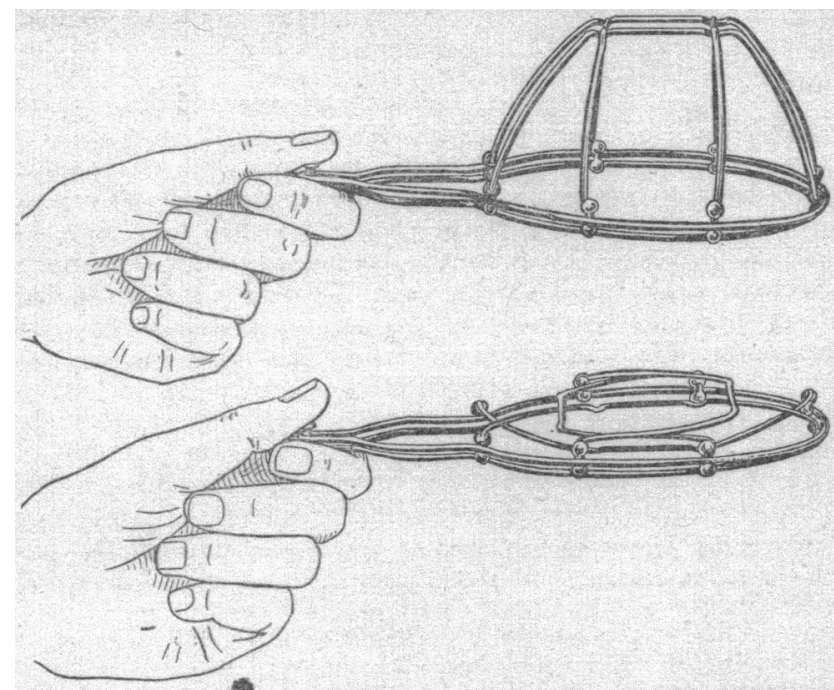

a flat instrument, which lends itself easily to packing into small compass. The modification was worked out owing to the fact that the ordinary surgical and obstetric bags contain too little room. Our modern armamentarium is bulky and economy of space is desirable. The instrument is simple, durable and compact.

\section{A SIMPLE STERILIZER FOR EMERGENCY AND COUNTRY WORK.* \\ EDWARD S. STEVENS, M.D. LEBANON, OHIO.}

It is one thing to prepare for operative work with the modern pressure sterilizer and other conveniences of the hospital; it is quite another thing to prepare for the same work in a private home, perhaps on a farm at a distance from the operator's office. The device offered is purposely presented in a crude form to show what may be done under such circumstances when the element of time is important. An instrument maker could give us a more sightly instrument. All that is required is a piece of No. 9 or $10^{\circ}$ wire, a sheet of wire netting, four cords for suspension and an equal number of metal hooks. In this case poultry netting was used, the cords were wire picture-cord, and the hooks were ordinary picture hooks. But any strong cord will answer and a hook may be speedily fashioned from sheet tin or iron.

The wire is shaped to fit loosely the inside of the house wash-boiler. The wire netting is bent and fastened over this frame, the cords are attached near the ends of the straight sides and are of such length as to let the apparatus into the boiler to within a few inches of the bottom; the hooks attached to the upper ends of the cords are placed over the upper rim of the boiler. This gives a steam sterilizer with considerable space. Boilers are usually found in one of three sizes-most commonly the No. 8.

- Presented at the January meeting of the Warren County (Ohio) Medical Society.
In using this simple apparatus a few inches of boiling water are placed in the boiler, the sterilizer is hooked over the rim of the boiler, and dressings, bedding and towels may then be steamed as long as may be desired. To prevent the condensation of the steam in the sterilized articles they may be placed for a few minutes in the oven, when they will be found to be dry enough for use.

I have found this little apparatus a great time-saver

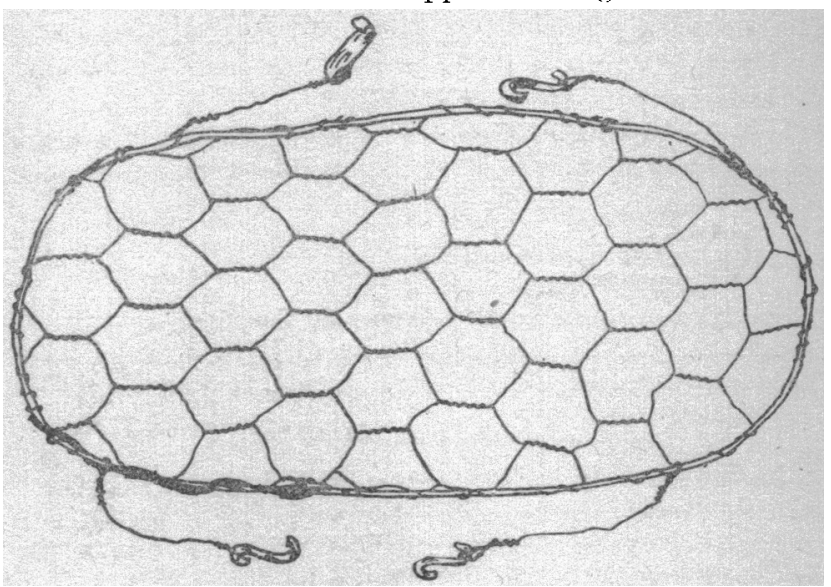

when called on for work which allowed little time for preparation. It is also a convenient article for the nurse who is sent ahead to prepare for the coming of the surgeon. By folding the cords and hooks over the wire netting it may be packed flat and is easily carried.

\section{AN IMPROVED PETRI DISH HOLDER.*}

HAROLD O. RUH.

Assistant in Pathology, Indiana University. BLOOMINGTON, IND.

Any one who has attempted bacteriologic work, involving the use of a large number of Petri dishes, has regretted the lack of a satisfactory Petri dish holder. No holder, satisfactory for the various procedures of sterilization, storage, plating and incubation is on the
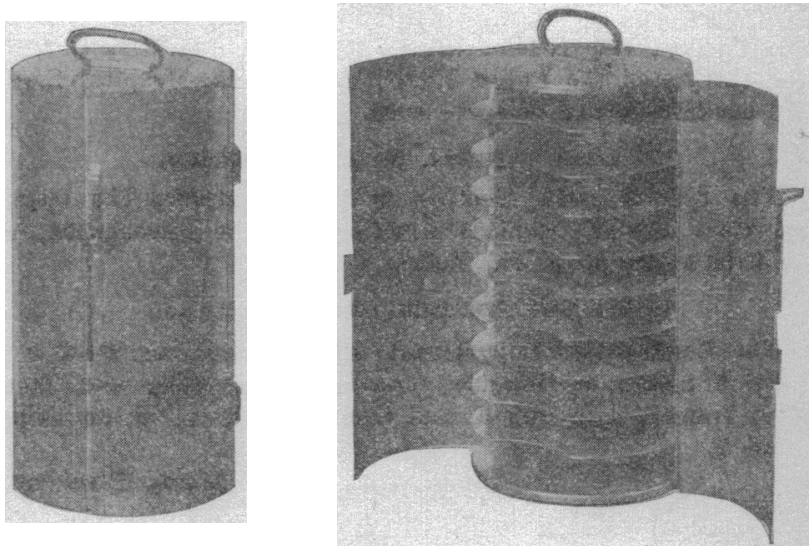

market. To supply this defect there has been designed the holder here presented.

The figure is self-explanatory. The instrument is made of heavy tin, galvanized iron, or other material that will withstand an ordinary amount of heat and moisture, and is made with pressed joints, without solder. The apparatus has been found satisfactory, so far as tested.

* From the Pathological Laboratory of Indiana University. 